\title{
Impact of attachment behavior on the treatment process of chronic pain patients
}

This article was published in the following Dove Press journal:

Journal of Pain Research

\author{
Ann-Christin Pfeifer ${ }^{1,2}$ \\ Juan Martin Gómez \\ Penedo ${ }^{3}$ \\ Johannes C Ehrenthal ${ }^{2}$ \\ Eva Neubauer' \\ Dorothee Amelung ${ }^{4}$ \\ Corinna Schroeter' \\ Marcus Schiltenwolf' \\ 'Department of Orthopedics, \\ Trauma Surgery and Paraplegiology, \\ Heidelberg University Hospital, \\ Heidelberg, Germany; ${ }^{2}$ Institute \\ of Medical Psychology, Center for \\ Psychosocial Medicine, University \\ Hospital Heidelberg, Heidelberg, \\ Germany; ${ }^{3}$ CONICET and Universidad \\ de Buenos Aires, Buenos Aires, \\ Argentina; ${ }^{4}$ School of Health Sciences, \\ University of Surrey, Guildford, UK
}

Background: Insecure attachment patterns are related to the onset and development of chronic pain. However, it is less documented on how short- and long-term effects of pain therapy might differ with the attachment style in interaction with specific pain conditions. We therefore examined how two different groups of chronic pain patients differ in their treatment trajectories and in regard to attachment.

Method: N=85/76/67 (T1/T2/T3) patients with medically unexplained musculoskeletal pain (UMP group) were compared to $n=89 / 76 / 56$ patients with joint pain from osteoarthritis (OA group), using multilevel modeling. UMP patients received a multimodal pain program, and OA patients received surgery. Pain intensity before (T1) and after (T2) treatment and at a 6 months follow-up (T3) was assessed by using a visual analog scale of pain.

Results: Pain patients report a significant reduction in pain intensity upon the completion of the treatment compared to T1. Over the next 6 months, the pain intensity has further declined for patients with low attachment anxiety. In contrast, patients with highly anxious attachment report an increase in pain intensity. This main effect of anxious attachment on pain is significant when predicting changes both in acute treatment and during follow-up while controlling for group effect. In addition, there is also an interactive effect of group by avoidant attachment. In the UMP group, high scores in avoidant attachment were associated with the lower reduction in pain severity, while in the OA group, high scores in attachment avoidance were associated with a steeper reduction in pain severity. Conclusion: The results indicate that insecurely attached patients with pain symptoms only benefit from a multimodal pain therapy in limited ways in regard to posttreatment trajectories. Maintaining positive results over a period of 6 months is a challenge, compared with securely attached patients. Significance: The results of this study suggest the importance of direct and indirect mechanisms of attachment and its relevance for the management of pain experiences. Therefore, to include the individual attachment patterns in the treatment may be a promising way to enhance the treatment prospects.

Keywords: attachment theory, chronic pain, multimodal treatment

\section{Background}

Patients' attachment patterns have been linked to the development and course of chronic pain conditions. ${ }^{1-5}$ Attachment may also play a differential role with regard to different subgroups of pain patients. Insecure attachment is more prevalent in patients suffering from chronic pain without clear organic cause (ie, somatoform disorders) than in patients diagnosed with osteoarthritis (OA). ${ }^{6}$

According to the Attachment-Diathesis Model of Chronic Pain, dysfunctional behavioral responses to acute pain lead to complication and chronification of conditions regardless of diagnosis. ${ }^{1}$
Correspondence: Ann-Christin Pfeifer Department of Orthopedics, Trauma Surgery and Paraplegiology, Heidelberg University Hospital, Schlierbacher Landstr. 200a, 69II8 Heidelberg, Germany

Tel +49622I 5635492

Email ann-christin.pfeifer@med.uniheidelberg.de 
However, while an insecure attachment pattern may not be the driving factor behind the development of OA, insecurely attached OA patients might be at a higher risk of developing chronic pain conditions because of inadequate reactions to acute pain such as inadequate coping mechanisms, lower stress resilience, more conflicting interactions with health care personnel, or lower compliance with the therapeutic goals. $^{7-10}$

However, empirical studies examining the effect of insecure attachment on the trajectory of pain conditions with and without clearly defined organic cause for patients in treatment are scarce. While several studies link insecure attachment with worse trajectories in multimodal pain treatments, ${ }^{1,11,12}$ with an exception for the precursor of this study, which was cross sectional only, none of them clearly differentiate between patients with and without clearly defined organic cause. ${ }^{6}$ The present study therefore seeks to answer the question whether or not patients with insecure attachment are less likely to obtain therapeutic gains during treatment and maintain them during follow-up. We hope that this study might help to close the aforementioned gap in attachment research with regard to chronic pain and pave a way toward differential treatment indications for patients based on the type of chronic pain condition and attachment. Thus, the core aim of the present study was to contribute to the understanding of potential factors that influence the treatment outcome of chronic pain patients participating in a multimodal pain program. For this purpose, we compared two different pain subgroups, one with (OA) and one without (unexplained musculoskeletal pain [UMP]) a clearly defined organic cause, in order to identify treatment of possible interaction effect of attachment with the type of chronic pain condition and independent of organic causes. The main questions of this study are: 1) Do insecurely attached pain patients differ from securely attached patients in their treatment trajectories from baseline to 6 months follow-up? 2) Are results similar for OA patients who undergo hip joint replacement and patients without clearly defined organic cause who take part in a 4-week multimodal pain program?

\section{Methods}

\section{Participants}

Participants were 174 adult patients, presenting UMP $(n=85)$ or pain from OA $(n=89)$. Patients of both the clinical samples were recruited in the Department of Orthopedic Surgery of the University of Heidelberg between September 2008 and January 2010. This research was approved by the University of Heidelberg ethics committee, and all patients provided written informed consent. Inclusion criterion for both samples was the patients should suffer from chronic musculoskeletal pain for at least 6 months. Exclusion criteria for both samples included insufficient ability to communicate in German, age $<18$ or $>80$ years, and a diagnosis of psychosis, bipolar disorder, or neurological disorder.

Patients from the OA group were significantly older and better educated at baseline compared to patients with medically unexplained pain. However, the two groups did not differ significantly with regard to reported pain intensity in the last 7 days or in their disability ratings prior to treatment. While the distinction between UMP and OA is not clear in day to day clinical practice, for the sake of eliminating most organic variables from the UMP group, comprehensive medical and psychological diagnostic procedures, including diagnostic imaging by an orthopedic specialist, were administered at intake in order to check whether pain was fully explained by a specific somatic pathology. See Schroeter et a ${ }^{6}$ for full details on baseline results and recruitment process. While there was a $29 \%$ drop out from $\mathrm{T} 1$ to $\mathrm{T} 3$, with $\mathrm{n}=85 / 76 / 67$ participating in the UMP group at T1, T2, and T3, respectively, and $\mathrm{n}=89 / 76 / 56$ for the OA group, the applied multilevel modeling technique has well accommodated the unbalanced data. ${ }^{13}$

\section{Treatments}

Patients in the UMP group received a multimodal pain treatment including an intensive, structured, full-time 4-week program, consisting of physiotherapy, psychotherapy, and medical treatment in the form of group and individual sessions. The treatment includes 6- to 7-hour program for 5 days a week. In 4 weeks, the patients in the UMP group received a total of 16 hours of individual psychotherapy offered by psychologists and doctors, 20 hours of Nordic walking, 4 hours of dance and music therapy, 8 hours of relaxation training, 8 hours of group therapy as well as 4 hours of a discussion group lead by medical doctors, 12 hours of group physiotherapy with an additional 8 hours of training in a gymnasium, and 6 hours of individual physiotherapy during their 4-week program. Furthermore, patients can use sporting equipment in the gymnasium for additional physical training on their own during free time slots after receiving a detailed introduction session by physiotherapists. Patients in the OA group underwent a hip joint replacement at the participating hospital. The present study did not examine the medication level, as patients from the UMP group were asked to reduce their individual medication over the course of the treatment but had severely different levels of medication type and dosage coming into treatment, making a coherent assessment 
of medication difficult, while also not being a focus of the present study. Patients from the OA group received their standard dosage of medication after the surgery.

\section{Measures}

\section{Primary outcome measures}

Pain during the present day and during the previous week was assessed using two visual analog scales (VASs) with scores ranging from 0 to 100 . Patients were also asked to indicate the number of days they experienced pain, and the number of days they experienced strong pain, both within the previous month. ${ }^{14,15}$

\section{Attachment style}

Patients' attachment patterns were assessed by the German versions of the Relationship Questionnaire (RQ-2) ${ }^{16}$ and the revised Experience of Close Relationships Questionnaire (ECR-R). ${ }^{17}$ We chose to include both the measures in order to assess generalized as well as relationship-specific attachment expectations.

The RQ-2 captures a person's dominant, cognitive schemata of self and others. It consists of four short paragraphs, each describing one attachment pattern (eg, the avoidant prototype reads, "I am comfortable without close emotional relationships. It is very important to me to feel independent and self-sufficient, and I prefer not to depend on others or have others depend on me"). The participants are then asked to rate their degree of correspondence to each of the prototypes on a 7-point Likert-type scale ranging from 1 (agree strongly) to 7 (disagree strongly). The scores associated with each prototype were used as a dimensional continuous measure for that attachment style.

The RQ-2 has acceptable psychometric properties, and it is relatively independent from self-deceptive biases. ${ }^{18-21}$ The RQ-2 has been implemented in cross-cultural studies as well. ${ }^{21}$ For categorical descriptive data of patient attachment, we used the labels stemming from the RQ-2, namely secure, dismissing, preoccupied, and fearful.

The ECR-R assesses the two regulatory attachment strategies: avoidance and anxiety. The instrument has been developed on the principles of item response theory and specifically assesses attachment styles in romantic relationships (eg, attachment anxiety: "I find that my partner/ partners doesn't/do not want to get as close as I would like;" attachment avoidance: "I find it difficult to allow myself to depend on romantic partners"). All items are rated on a scale from 1 to 7 , and the mean values are computed for the two scales.
The ECR-R has very good internal consistency, a stable factor structure, high test-retest reliability, and construct validity. ${ }^{22}$ Psychometric properties are comparable in the German version, ${ }^{23}$ and the questionnaire is successfully applied in a wide spectrum of psychiatric and medical studies. ${ }^{24,25}$

In all analyses we used sum scores for the anxious and avoidant attachment scales of the ECR-R, as well as a composite score consisting of the sum of the anxious and avoidant scale together, to get a measure of general insecurity. In this, patients with one SD above the mean in general insecurity were labeled insecure, for the purpose of the hierarchical linear modeling (HLM) analysis, while patients with general insecurity one level below the mean were labeled secure. The attachment styles measured by the ECR-R were treated as continuous variables.

\section{Procedures}

Measurements were performed in both groups before the treatment ( $\mathrm{t} 1)$, immediately after the treatment ( $\mathrm{t} 2)$, and at a 6-month follow-up (t3).

\section{Analytic strategy}

For the analysis of the longitudinal data, we used HLM analysis ${ }^{26}$ as it addresses the expected dependency in longitudinal data. Furthermore, as HLM uses both individual and sample information to estimate the parameters, it deals with missingness in data at the repeated measures level (ie, at level 1). Every case that has a score in the outcome measures at least one of the time points was included in the analysis, mimicking an intent-to-treat approach. In all models applied, measurements over time in the outcome variables (level 1) were nested in patients (level 2). Time was treated as continuous variable, measured in weeks $(\mathrm{t} 1=0$ weeks, $\mathrm{t} 2=4$ weeks, $\mathrm{t} 3=24$ weeks). More specifically, for this study we conducted a piecewise, two-level growth curve model, estimating within-subjects weekly rate of change during treatment and follow-up period, and between-subjects differences in both pieces. ${ }^{26}$ The first piece estimates the change observed between $\mathrm{T} 1$ and $\mathrm{T} 2$ (ie, acute treatment), while the second piece estimates the change observed between $\mathrm{T} 2$ and T3 (ie, follow-up period).

We decided to use this piecewise approach since the outcome trajectories might be different during treatment, where a more pronounced reduction is expected vs during follow-up, where a less steep reduction or even deterioration might be expected.

Although for longitudinal analysis, the best option is to have at least three time points at each piece of the model, ${ }^{13}$ it is also possible to estimate the change having only two 
time points in each. ${ }^{27}$ However, in order to be able to run these analyses, it is necessary to provide extra information for the models. ${ }^{27,28}$ Thus, we calculated and included the estimated error variance at level 1 for the outcome measures in the model. The level 1 error variance of each outcome measure was estimated as the product of its measurement error (1-Cronbach's $\alpha$ ) and the variance of the measure at each time point.

When testing multiple hypotheses, as with the different attachment measures, type I error rate was addressed by estimating the number of true null hypothesis prior to adopting false discovery rate control. ${ }^{29}$

We first ran an unconditional baseline model with time as the only predictor at level 1 . Then, we ran another unconditional model including linear slope for piece 1 and the linear slope for piece 2 as predictors at level 1. Time was centered at week 4 (t2), the midpoint in time between piece 1 and piece 2 . Thus, in those later models, the intercept represents the level of severity at week 4 , while the two slope parameters represent the weekly rate of change during treatment and follow-up. For full model equations, see Supplementary materials.

Next, to answer the question of whether belonging to either the UMP or the OA group has a significant effect on the outcome, we conducted conditional models including the dummy coded group variable $(\mathrm{UMP}=0 ; \mathrm{OA}=1)$ as a level 2 predictor of the pain severity level at week 4 , the weekly rate of change during treatment, and the weekly rate of change during follow-up.

Then, to test a possible impact of general attachment insecurity on pain, we tested conditional models including both the groups ( $\mathrm{UMC}=0 ; \mathrm{OA}=1$ ) and grand mean centered scores in the attachment measures as level 2 predictors. These models (one for each attachment index, ie, RQ-2 and ECR-R) allow us to identify between-subject effects of attachment indexes, controlling for group effects.
Finally, to test for a differential effect of attachment on the outcome by group, we added interaction effects of group with attachment indexes as predictors of the models. Prior to the creation of the interactive terms, as suggested by Aiken et $\mathrm{al},{ }^{30}$ the attachment indexes were grand mean centered.

The data has $7.05 \%$ of missing values, and $26.5 \%$ of cases included one or more missing values. While multilevel models cope well with unbalanced designs, all the analyses were performed under the assumption that observations are missing at random (MAR), implying that missingness is not related to the dependent variable at dropout. ${ }^{31}$ For all descriptive analyses, missing values were imputed by multiple imputations of chained equations, ${ }^{32}$ which gives unbiased estimates of the data under MAR assumptions.

SPSS Version 22 (IBM Corporation, Armonk, NY, USA) was used for preliminary analysis and data management while the HLM7 software was used for multilevel modeling.

\section{Results \\ Descriptives}

\section{Prevalence of an insecure attachment style}

The frequencies of global RQ-2 attachment style categories are presented in Table 1 and the proportions are displayed in Figure 1. Pain conditions differed significantly according to attachment security (chi-squared test, $\chi^{2}(1)=11.24$, $P=0.002)$. About one-third of UMP patients $(38.8 \%)$ and two-thirds of OA patients were classified as securely attached.

\section{Unconditional models}

As expected, when comparing the two unconditional models with time as the only predictor, the piecewise model better fit data than the model with a single linear slope, $\chi^{2}(4)=139.26, P<0.001$.

Results of the piecewise model indicated that on average, patients presented a significantly lower pain severity level

Table I Descriptives of core study variables

\begin{tabular}{|c|c|c|c|c|c|c|}
\hline & \multicolumn{2}{|c|}{ UMP (n=85) } & \multicolumn{2}{|c|}{ OA $(n=89)$} & \multirow[t]{2}{*}{ Statistical test } & \multirow[t]{2}{*}{$P$-value } \\
\hline & $M / \%$ & SD & $M / \%$ & SD & & \\
\hline Age, years & 48.87 & II.57 & 57.98 & 11.06 & $\mathrm{t}_{(170.6)}=5.30$ & $<0.001$ \\
\hline ECR attachment anxiety & 2.43 & 1.05 & 2.16 & 1.06 & $\mathrm{t}_{(171.7)}=1.67$ & 0.09 \\
\hline ECR attachment avoidance & 2.4 & 1.12 & 2.21 & 1.06 & $\mathrm{t}_{(170.2)}=\mathrm{I} .17$ & 0.24 \\
\hline Initial Pain & 60.26 & 20.12 & 60.8 & 26.11 & $t_{(166.6)}=-0.15$ & 0.88 \\
\hline Female & $67 \%$ & - & $55 \%$ & - & $\chi_{(1)}^{2}=3.12$ & 0.07 \\
\hline RQ secure & $41 \%$ & - & $77 \%$ & - & $\chi_{(1)}^{2}=22.4$ & $<0.001$ \\
\hline RQ enmeshed & $14 \%$ & - & $4 \%$ & - & $\chi_{(1)}^{2}=3.73$ & 0.053 \\
\hline RQ preoccupied avoidant & $28 \%$ & - & $16 \%$ & - & $\chi_{(1)}^{2}=3.92$ & 0.047 \\
\hline $\mathrm{RQ}$ anxious avoidant & $16 \%$ & - & $5 \%$ & - & $\chi_{(1)}^{2}=4.2$ & 0.04 \\
\hline
\end{tabular}

Note: Bold entries represent differences significant at at least $P<0.05$.

Abbreviations: UMP, unexplained musculoskeletal pain; OA, osteoarthritis; ECR, Experience of Close Relationships Questionnaire; RQ, Relationship Questionnaire. 


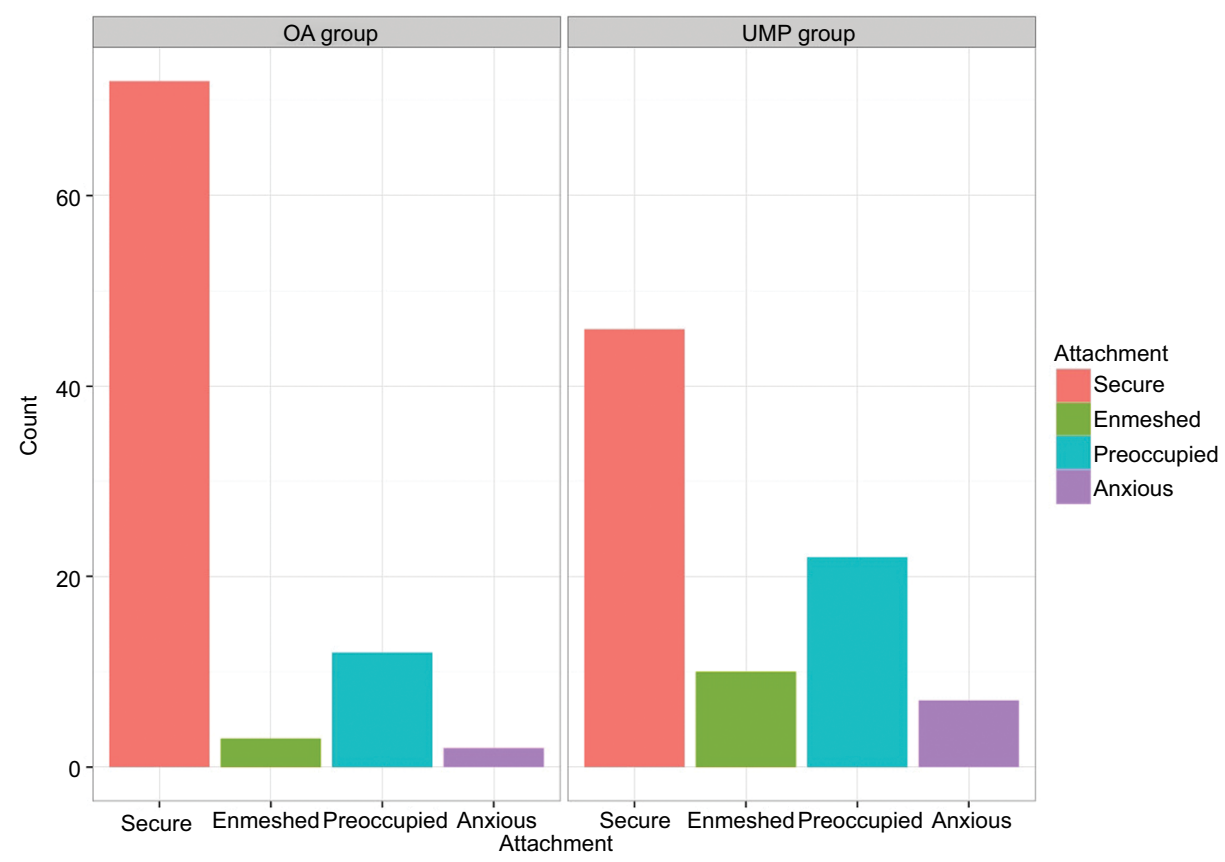

Figure I Counts of attachment style per group pretreatment.

Abbreviations: UMP, unexplained musculoskeletal pain; OA, osteoarthritis.

of 31.67 , with a mean change of $29.10(\mathrm{SE}=1.98, \mathrm{t}(173)$ $=14.708, P<0.001)$ in contrast to the average pretreatment pain level of 60.82 . Moreover, on average, patients had a significant weekly reduction of pain severity during treatment $(\beta=-4.63, \mathrm{SE}=0.45, \mathrm{t}(173)=-10.264, P<0.001)$. There was no significant change in pain severity after discharge $(\beta=0.03$, $\mathrm{SE}=0.07, \mathrm{t}(173)=0.502, P=0.62)$.

\section{Conditional group effects model}

The main effects model including the group as a level 2 predictor shows that the participants significantly differed in their levels of pain severity at the end of treatment $(\beta=-26.18$, SE $=3.42, P<0.001)$ and in their weekly rate of change in pain severity during treatment $(\beta=-3.06, \mathrm{SE}=0.86, P<0.001)$. Patients with OA had lower levels of pain severity at treatment termination and a greater rate of change during the treatment, compared with patients with UMP. However, the two groups did not significantly differ on the weekly rate of change in pain severity during the follow-up period $(\beta=-0.11$, $\mathrm{SE}=0.14, P=0.43$ ).

\section{Conditional attachment effect models}

In Table 2, we presented all the models including attachment indexes and group as level 2 predictors. We only observed one index of attachment having a significant effect on pain severity. Regardless of the group of the participants, insecure attachment measured by the ECR-R presented a significant main effect on patients' weekly rate of pain severity reduction both during treatment $(\beta=-0.05, \mathrm{SE}=0.02, \mathrm{t}(156)=-2.035$, $P=0.04)$ and during follow-up $(\beta=0.01, \mathrm{SE}=0.003, t(156)$ $=2.069, P=0.04)$. Patients with higher insecure attachment showed greater reductions in pain severity during treatment, but also greater deterioration during follow-up (Figure 2).

There was no significant effect of insecure attachment on pain severity levels at posttreatment $(\beta=-0.06, \mathrm{SE}=0.09$, $t(156)=-0.650, P<0.52)$.

\section{Interaction effects}

Column 4 of Table 2 shows the interaction effects of specific attachment by group on treatment severity. From the models conducted, we found a significant interaction effect of avoidant attachment by group on the weekly rate of change during treatment $(\beta=-1.09, \mathrm{SE}=0.44, t(163)=-2.454, P=0.02)$. While patients in the UMP group with low avoidant attachment had a greater reduction of pain severity during treatment than patients with higher avoidant attachment, in the OA group the patients with lower avoidant attachment had a slightly less steep reduction in pain severity in comparison with patients with higher avoidant attachment (Figure 3). However, there was no significant interaction effect of avoidant attachment by group on the level of pain severity at posttreatment $(\beta=-2.81, \mathrm{SE}=1.77, \mathrm{t}(163)=-1.582, P=0.12)$, or 
Table 2 Parameter estimates for HLM models

\begin{tabular}{|c|c|c|c|c|c|c|c|c|c|c|}
\hline \multirow[t]{2}{*}{ DV: severe pain } & \multicolumn{3}{|c|}{ Unconditional model } & \multicolumn{3}{|c|}{ Group as main effect } & \multicolumn{3}{|c|}{ Fully conditional model } & \multirow{2}{*}{$\begin{array}{l}\text { Attachment } \\
\text { by group } \\
\text { B }\end{array}$} \\
\hline & B & SE & $P$-value & $\mathbf{B}$ & SE & $P$ P-value & $\mathbf{B}$ & SE & $P$-value & \\
\hline \multicolumn{11}{|l|}{ Fixed parts } \\
\hline$(\text { Intercept })_{\mathrm{T} 0}$ & 29.10 & 1.98 & $<0.001$ & 42.34 & 2.44 & $<0.001$ & 41.98 & 2.51 & $<0.001$ & 42.62 \\
\hline Group $_{\mathrm{T} 0}$ & & & & -26.18 & 3.42 & $<0.001$ & -25.02 & 3.60 & $<0.001$ & -26.49 \\
\hline Insecure $_{\mathrm{T} 0}$ & & & & & & & -0.06 & 0.09 & 0.517 & \\
\hline Avoidance $_{\mathrm{T} 0}$ & & & & & & & & & & 1.95 \\
\hline Avoidance $\times$ group $_{\mathrm{T} 0}$ & & & & & & & & & & -2.80 \\
\hline$\overline{(\text { Intercept })_{\mathrm{TI}}}$ & -4.63 & 0.45 & $<0.001$ & -3.10 & 0.61 & $<0.001$ & -3.20 & 0.62 & $<0.001$ & 41.98 \\
\hline Group $_{\mathrm{TI}}$ & & & & -3.06 & 0.86 & $<0.001$ & -2.91 & 0.88 & $<0.001$ & -25.02 \\
\hline Insecure $_{\mathrm{TI}}$ & & & & & & & -0.05 & 0.02 & 0.044 & \\
\hline Avoidance $_{\mathrm{TO}}$ & & & & & & & & & & 0.62 \\
\hline Avoidance $\times$ group $_{\mathrm{TO}}$ & & & & & & & & & & -1.09 \\
\hline (Intercept) $)_{\mathrm{T} 2}$ & 0.03 & 0.07 & 0.617 & 0.08 & 0.09 & 0.406 & 0.07 & 0.10 & 0.464 & 41.98 \\
\hline Group $_{\mathrm{T} 2}$ & & & & -0.11 & 0.14 & 0.430 & -0.07 & 0.14 & 0.604 & -25.02 \\
\hline Insecure $_{\mathrm{T} 2}$ & & & & & & & 0.01 & 0.00 & 0.040 & \\
\hline Avoidance $_{\text {T0 }}$ & & & & & & & & & & -0.02 \\
\hline Avoidance $\times$ group $_{\mathrm{T} 0}$ & & & & & & & & & & 0.08 \\
\hline \multicolumn{11}{|l|}{ Random parts } \\
\hline$\sigma^{2}$ & 332.40 & & & 332.45 & & & 332.10 & & & 331.85 \\
\hline$\tau_{1 \mathrm{IITI}}$ & 46.58 & & & 26.97 & & & 24.96 & & & 23.9 \\
\hline $\mathrm{ICC}_{\mathrm{TI}}$ & 0.73 & & & 0.64 & & & 0.64 & & & 0.62 \\
\hline$\tau_{12, \mathrm{~T} 2}$ & 1.31 & & & 0.49 & & & 0.80 & & & 0.84 \\
\hline $\mathrm{ICC}_{\mathrm{T} 2}$ & 0.34 & & & 0.28 & & & 0.26 & & & 0.25 \\
\hline
\end{tabular}

Notes: $P$-values for random effects were computed using parametric bootstrap.

Abbreviations: DV, dependent variable; SE, standard error; $\sigma^{2}$, residual variance; $\tau$, random intercept; ICC, intraclass correlation coefficient.

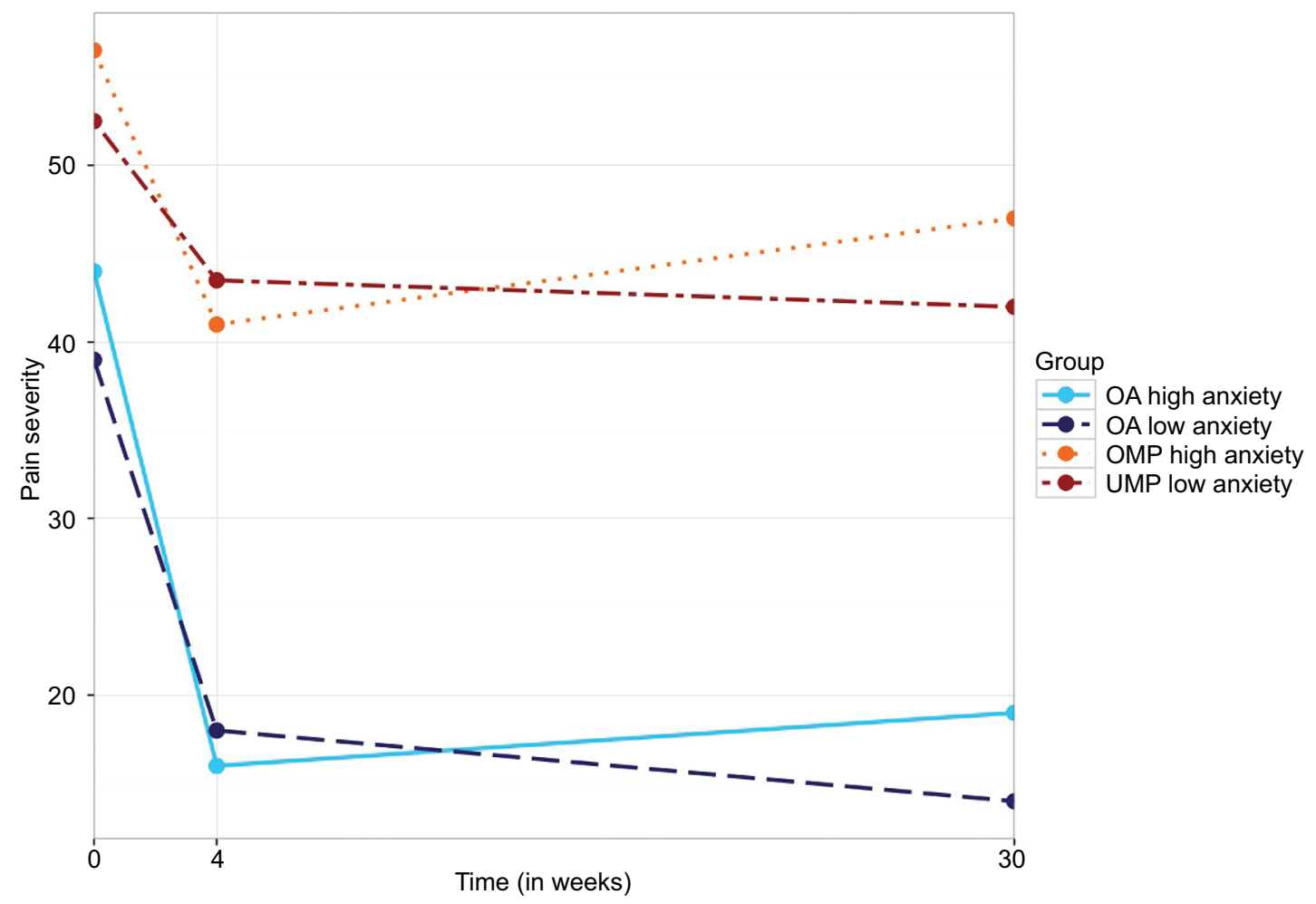

Figure 2 Interaction effect of pain severity over time with anxious attachment per group.

Abbreviations: UMP, unexplained musculoskeletal pain; OA, osteoarthritis. 


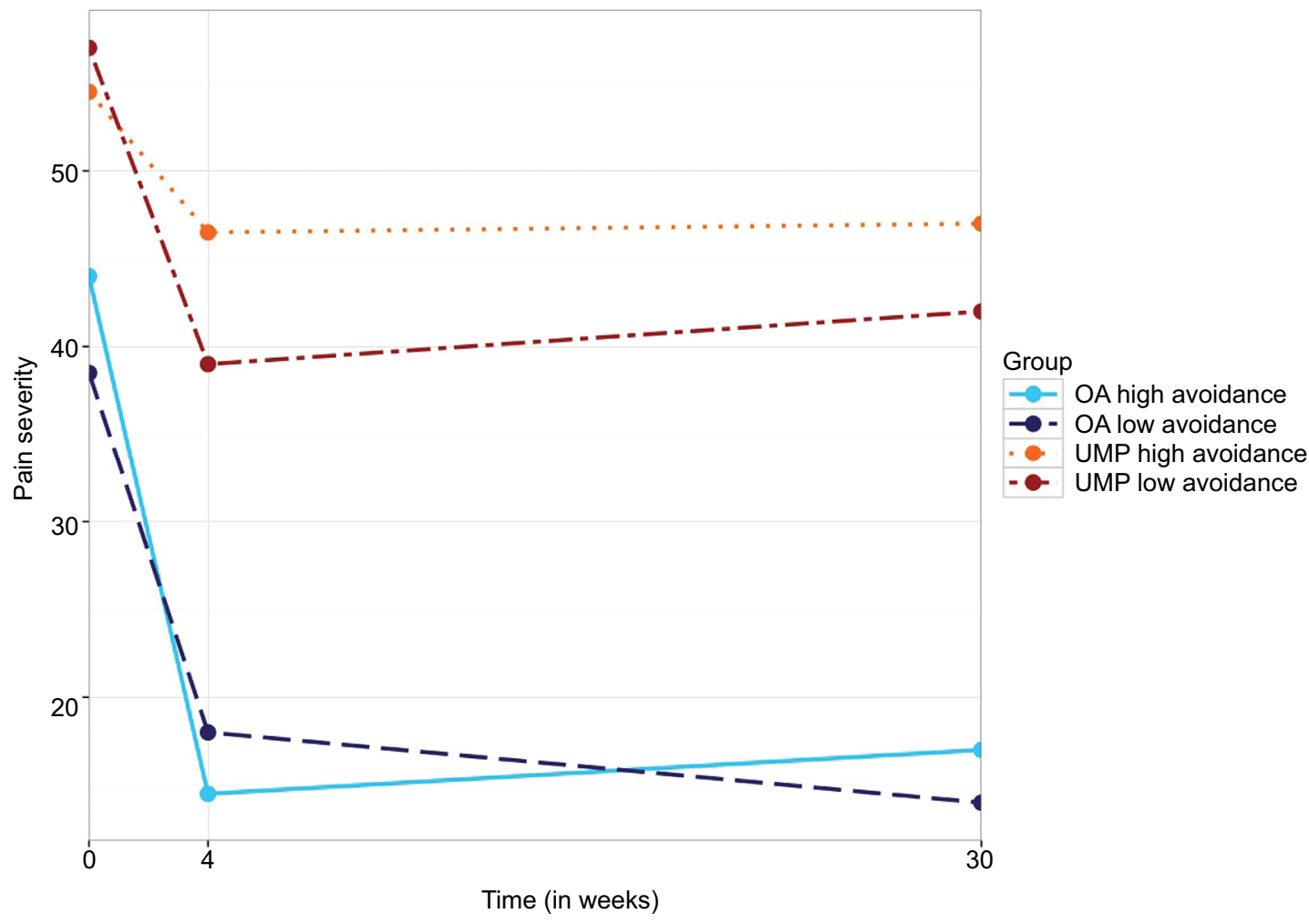

Figure 3 Interaction effect of pain severity over time with avoidant attachment per group. Abbreviations: UMP, unexplained musculoskeletal pain; OA, osteoarthritis.

in the weekly rate of change during follow-up $(\beta=0.08$, SE $=0.07, \mathrm{t}(163)=1.146, P=.25)$. For a table of all HLM results, see Supplementary materials.

\section{Discussion}

Consistent with the past research on attachment and pain, prevalence of an insecure attachment pattern was significantly higher in the UMP group compared to the OA group. ${ }^{12,33,34}$ About two thirds of the patients of the UMP group showed an insecure attachment pattern, whereas only one third of the OA group reported one of the insecure attachment styles which equals the prevalence of a healthy representative population sample. ${ }^{35,36}$

Both the patient groups reported a marked decrease in average pain intensity at T2 after discharge from hospital having either completed surgery or the pain therapy. The group predictor, indicating the different pain conditions, achieved the highest effect size in the model which might be explained by the different treatments that both groups had received. While patients in the OA group had a clear somatic diagnosis and received the standard treatment for it, patients in the UMP group did not have a clear diagnosis, making treatment much more difficult.
That being said, time was a significant predictor independent from group which indicates that both the treatments work in reducing pain severity (Table 2 , column 1 ). However, both the treatments do not retain their effects after the treatment duration is over, possibly because patients struggle to manage their pain by themselves again without the help of a professional team. ${ }^{12,37,38}$

Kolb compares this first reaction to the treatment as form of placebo effect which is triggered by the attachment behavior of the patients. ${ }^{37}$

In the present study, average pain levels decreased equally for all patients regardless of their attachment style right after discharge. However, the findings of the present study also indicate that pain patients with various attachment patterns show different treatment outcomes on the long term. While the pain level further declined in patients with lower attachment anxiety, the pain level increased again in patients with high attachment anxiety at the 6 months follow-up. It is possible that patients with an insecure attachment style report higher levels of pain after losing the comfort and safety of support and help from a multidisciplinary health care team. Generally, secure attachment is associated with a support system as securely attached people have very close members 
they can confide in and rely on, whereas insecurely attached people have no or insufficient support available to them. ${ }^{39}$ This could explain that the insecurely attached (anxiously and avoidant attached) patients were able to benefit from the pain therapy in the same way as the securely attached patients as the multidisciplinary team was able to provide the support system while undergoing the treatment. However, following this train of thought, as soon as the treatment was over and the support system unavailable, these insecure patients lost their "secure base" leading to an increase in pain. The patients then had to experience their pain again without the help of others which can be seen as threatening especially by the insecurely attached patients who tend not to have a sufficient support system. ${ }^{40}$ It is well documented in the literature that insecurely attached patients do not comply with treatments to the same level as securely attached patients. ${ }^{7-10}$ It has also been proposed that patients with insecure attachment are prone to experience poorer pain-related adjustments once chronic pain has already manifested. ${ }^{12,41}$ This could offer another explanation as to why insecure patients benefited less from the treatment on a long term. Securely attached patients seemed to be better in using the learned techniques and comply with the treatment concept even after the end of the treatment. ${ }^{42}$ The model also showed that being in the UMP group was associated with higher pain levels across different time points. This is in line with the findings from other studies pointing out that patients who suffer from somatoform pain disorders have an increased pain perception compared to the general population. ${ }^{43}$ The reason for that could be that patients with medically unexplained pain have a body that is more sensitive and might tend to worsen the pain. ${ }^{11}$

\section{Limitations}

The biggest limitation of the present study lies in the comparability of the two treatment groups. Treatment of the two subsamples varied in length of the treatment and type of the treatment. The OA group received surgery and, therefore, had an average stay in hospital for about 10 days, whereas, the UMP group participated in a 4-week multidisciplinary pain treatment program which included group and individual psychotherapy as well as physiotherapy. The concepts of both the types of treatment are very different, not only in the physiological vs psychological approach but also in terms of supportive medication. Patients who received surgery were given standard dosage of pain medication afterwards, while the multidisciplinary pain treatment program tried to reduce the medication usage of the patients. This could have had an effect on the perceived pain levels of the patients, possibly contributing toward the differ- ence in pain levels between the treatments. Also, the use of a VAS for the main outcome instead of a questionnaire assessing different dimensions of pain, while ecological, is a shortcoming of the present study. Also, while the interaction effects of attachment indicate insecure patients not being able to retain their gains during treatment for a longer period of time, the follow-up in the present study was only of 6 months in length. This effect might be more pronounced in longer follow-ups and may the high treatment frequencies seen in chronic pain patients, but in order to test this hypothesis, a study with a longer follow-up is needed. Additionally, the fact that the interaction patterns, especially in the case of the interaction of attachment anxiety by treatment, show similar trajectories despite the very different treatment modalities might hint at the generalizability of these results onto other treatments.

\section{Conclusion}

The present study provides estimates of the effects of attachment on average pain intensity in two different samples of pain patients. Pain patients were found to report reduced pain level after discharge from either hospital after surgery or an outpatient pain treatment program. However, insecurely attached patients were less able to maintain the positive results of the treatment over a period of time. These results indicate that, in pain patients, attachment seems to be having a pronounced impact on treatment outcomes until 6 months after the termination of treatment and possibly longer. Therefore, an attachment-based approach may be a promising way to enhance the prospects especially for patients who suffer from medically unexplained pain.

\section{Acknowledgments}

The authors gratefully acknowledge the support of the staff and clients of the Department of Orthopedics, Trauma Surgery, and Paraplegiology at Heidelberg University Hospital. We would like to thank the private foundation "Psychosomatik der Wirbelsäulenerkrankungen" ("Psychosomatics of spine disorders") for funding this work. The funders had no role in study design, data collection and analysis, decision to publish, or preparation of the manuscript.

\section{Author contributions}

All authors contributed toward data analysis, drafting and revising the paper, gave final approval of the version to be published, and agree to be accountable for all aspects of the work.

\section{Disclosure}

The authors report no conflicts of interest in this work. 


\section{References}

1. Meredith P, Ownsworth T, Strong J. A review of the evidence linking adult attachment theory and chronic pain: presenting a conceptual model. Clin Psychol Rev. 2008;28(3):407-429.

2. Porter LS, Davis D, Keefe FJ. Attachment and pain: recent findings and future directions. Pain. 2007;128(3):195-198.

3. Waller E, Scheidt CE, Hartmann A. Attachment representation and illness behavior in somatoform disorders. JNerv Ment Dis. 2004;192(3):200-209.

4. Hunter J, Maunder R, editors. Advanced concepts in attachment theory and their application to health care. In: Improving Patient Treatment with Attachment Theory. Switzerland: Springer International Publishing; 2016:27-37.

5. Maunder RG, Hunter JJ. Attachment and psychosomatic medicine: developmental contributions to stress and disease. Psychosom Med. 2001;63(4):556-567.

6. Schroeter C, Ehrenthal JC, Giulini M, et al. Attachment, symptom severity, and depression in medically unexplained musculoskeletal pain and osteoarthritis: a cross-sectional study. PLoS One. 2015;10(3):e0119052.

7. Bennett JK, Fuertes JN, Keitel M, Phillips R. The role of patient attachment and working alliance on patient adherence, satisfaction, and health-related quality of life in lupus treatment. Patient Educ Couns. 2011;85(1):53-59.

8. Ciechanowski PS, Katon WJ, Russo JE, Walker EA. The patient-provider relationship: attachment theory and adherence to treatment in diabetes. Am J Psychiatry. 2001;158(1):29-35.

9. Mikail SF, Henderson PR, Tasca GA. An interpersonally based model of chronic pain: an application of attachment theory. Clin Psychol Rev. 1994;14(1):1-16.

10. Obegi JH, Berant E. Attachment Theory and Research in Clinical Work with Adults. New York: Guilford Press; 2010.

11. Ciechanowski P, Sullivan M, Jensen M, Romano J, Summers H. The relationship of attachment style to depression, catastrophizing and health care utilization in patients with chronic pain. Pain. 2003;104(3):627-637.

12. Kowal J, McWilliams LA, Péloquin K, Wilson KG, Henderson PR, Fergusson DA. Attachment insecurity predicts responses to an interdisciplinary chronic pain rehabilitation program. J Behav Med. 2015;38(3):518-526.

13. Singer JD, Willett JB. Applied Longitudinal Data Analysis: Modeling Change and Event Occurrence. New York: Oxford University Press; 2003.

14. Carlsson AM. Assessment of chronic pain. I. Aspects of the reliability and validity of the visual analogue scale. Pain. 1983;16(1):87-101.

15. Bijur PE, Silver W, Gallagher EJ. Reliability of the visual analog scale for measurement of acute pain. Acad Emerg Med. 2001;8(12):1153-1157.

16. Bartholomew K, Horowitz LM. Attachment styles among young adults: a test of a four-category model. J Pers Soc Psychol. 1991;61(2):226-244.

17. Fraley RC, Waller NG, Brennan KA. An item response theory analysis of self-report measures of adult attachment. J Pers Soc Psychol. 2000;78(2):350-365.

18. Holtzman S, Delongis A. One day at a time: the impact of daily satisfaction with spouse responses on pain, negative affect and catastrophizing among individuals with rheumatoid arthritis. Pain. 2007;131(1-2):202-213.

19. Leak GK, Parsons CJ. The susceptibility of three attachment style measures to socially desirable responding. Soc Behav Pers. 2001;29(1):21-29.

20. Mestel R, von Wahlert J. Veränderungen der Bindungsstile von 6.800 Patienten während stationärer psychosomatischer Rehabilitation. Internetausgabe des Tagungsbandes. 2009;418. Available from: https:// frl.publisso.de/resource/frl:6008007-1/data\#page=419. Accessed September 14, 2018.

21. Schmitt DP, Alcalay L, Allensworth M, et al. Patterns and universals of adult romantic attachment across 62 cultural regions: are models of self and of other pancultural constructs? J Cross Cult Psychol. 2004;35(4):367-402.
22. Sibley CG, Fischer R, Liu JH. Reliability and validity of the revised experiences in close relationships (ECR-R) self-report measure of adult romantic attachment. Pers Soc Psychol Bull. 2005;31(11): 1524-1536.

23. Ehrenthal J, Dinger U, Lamla A, Funken B, Schauenburg H. Evaluation der deutschsprachigen Version des Bindungsfragebogens, Experiences in Close Relationships - Revised (ECR-RD) [Evaluation of the German Version of the Attachment Questionnaire, Experiences in Close Relationships - Revised (ECR-RD)]. Psychother Psychosom Med Psychol. 2009;59(6):215-223.

24. Ehrenthal JC, Friederich H-C, Schauenburg H. Separation recall: psychophysiological response-patterns in an attachment-related short-term stressor. Stress Health. 2011;27(3):251-255.

25. Manes S, Nodop S, Altmann U, et al. Social anxiety as a potential mediator of the association between attachment and depression. J Affect Disord. 2016;205:264-268.

26. Raudenbush SW, Bryk AS. Hierarchical Linear Models: Applications and Data Analysis Methods. Vol 1. Thousand Oaks, CA: Sage; 2002.

27. Smith JZ, Sayer AG, Goldberg AE. Multilevel modeling approaches to the study of LGBT-parent families: Methods for dyadic data analysis. In: Goldberg AE, Allen KR, editors. LGBT-Parent Families. New York, Springer; 2013:307-323.

28. Barnett RC, Marshall NL, Raudenbush SW, Brennan RT. Gender and the relationship between job experiences and psychological distress: a study of dual-earner couples. J Pers Soc Psychol. 1993;64(5): 794-806.

29. Benjamini Y, Hochberg Y. On the adaptive control of the false discovery rate in multiple testing with independent statistics. J Educ Behav Stat. 2000;25(1):60-83

30. Aiken LS, West SG, Reno RR. Multiple Regression: Testing and Interpreting Interactions. Thousand Oaks, CA: Sage; 1991.

31. Enders CK. Applied Missing Data Analysis. New York: The Guilford Press; 2010.

32. van Buuren G-O. Mice: multivariate imputation by chained equations in R. J Stat Softw. 2011;45(3):1-67.

33. Davies KA, MacFarlane GJ, McBeth J, Morriss R, Dickens C. Insecure attachment style is associated with chronic widespread pain. Pain. 2009;143(3):200-205.

34. Wang H, Weber A, Schiltenwolf M, Amelung D. Attachment style and cytokine levels in patients with fibromyalgia. A prospective longitudinal study. Schmerz. 2014;28(5):504-512.

35. Mickelson KD, Kessler RC, Shaver PR. Adult attachment in a nationally representative sample. J Pers Soc Psychol. 1997;73(5):1092-1106.

36. van Ijzendoorn MH, Kroonenberg PM. Cross-cultural patterns of attachment: a meta-analysis of the strange situation. Child Dev. 1988;59(1):147-156.

37. Kolb LC. Attachment behavior and pain complaints. Psychosomatics. 1982;23(4):413-425.

38. Merle C, Brendle S, Wang H, Streit MR, Gotterbarm T, Schiltenwolf M. Multidisciplinary treatment in patients with persistent pain following total hip and knee arthroplasty. J Arthroplasty. 2014;29(1):28-32.

39. Bifulco A, Moran PM, Ball C, Bernazzani O. Adult attachment style. I: its relationship to clinical depression. Soc Psychiatry Psychiatr Epidemiol. 2002;37(2):50-59.

40. Mikulincer M, Shaver PR. An attachment and behavioral systems perspective on social support. J Soc Pers Relat. 2009;26(1):7-19.

41. Meredith PJ, Strong J, Feeney JA. Evidence of a relationship between adult attachment variables and appraisals of chronic pain. Pain Res Manag. 2005;10(4):191-200.

42. Levy KN, Ellison WD, Scott LN, Bernecker SL, Style A. Attachment style. J Clin Psychol. 2011;67(2):193-203.

43. Dao TT, Leresche L. Gender differences in pain. J Orofac Pain. 2000;14(3):169-184; discussion 184-195. 


\section{Supplementary materials \\ Model equations for the hierarchical model analysis}

Unconditional model:

Level-1 model:

Pain severity $_{\mathrm{ij}}=\beta_{0 \mathrm{j}}+\beta_{1 \mathrm{j}} *\left(\right.$ Piece $\left.1_{\mathrm{ij}}\right)+\beta_{2 \mathrm{j}} *\left(\right.$ Piece $\left.2_{\mathrm{ij}}\right)+\mathrm{r}_{\mathrm{ij}}$

Level-2 model:

$$
\begin{aligned}
& \beta_{0 j}=\gamma_{00}+u_{0 j} \\
& \beta_{1 j}=\gamma_{10}+u_{1 j} \\
& \beta_{2 j}=\gamma_{20}+u_{2 j}
\end{aligned}
$$

where at level 1 the pain severity of patient $j$ at the moment $\mathrm{i}$ is estimated by patient j's pain severity at week $4\left(\beta_{0 \mathrm{j}}\right)$, weekly rate of change during treatment $\left(\beta_{1 j}\right)$, and weekly rate of change during follow-up $\left(\beta_{2 j}\right)$. The random effect at level $1\left(r_{i j}\right)$ allow the pain severity of the patient $j$ at moment $i$ to vary around the estimate score. At level 2 , the patient $j$ 's pain severity at week $4\left(\beta_{0 j}\right)$ is predicted by the average sample pain severity at week $4\left(\gamma_{00}\right)$, the weekly rate of change during treatment of patient $\mathrm{j}\left(\beta_{1 \mathrm{j}}\right)$ is predicted by the average weekly rate of change during treatment in the sample $\left(\gamma_{10}\right)$, and the weekly rate of change during follow-up $\left(\beta_{2 j}\right)$ is predicted by the average weekly rate of change during follow-up period in the complete sample. The random effects $\left(\mathrm{u}_{0 \mathrm{j}}, \mathrm{u}_{1 \mathrm{j}}\right.$, and $\left.\mathrm{u}_{2 \mathrm{j}}\right)$ allow the patient $\mathrm{j}$ to vary across the average intercept and slopes in the sample.

The final equation for the interactive models was as follows:

Level-1 model:

Pain severity ${ }_{\mathrm{ij}}=\beta_{0 \mathrm{j}}+\beta_{1 \mathrm{j}} *\left(\right.$ Piece $\left.1_{\mathrm{ij}}\right)+\beta_{2 \mathrm{j}} *\left(\right.$ Piece $\left.2_{\mathrm{ij}}\right)+\mathrm{r}_{\mathrm{ij}}$

Level 2 model:

$$
\begin{aligned}
& \beta_{0 \mathrm{j}}=\gamma_{00}+\beta_{01} *\left(\operatorname{Group}_{\mathrm{i}}\right)+\beta_{02} *\left(\text { Attachment index }_{\mathrm{i}}\right) \\
& +\beta_{03} *\left(\text { Interaction }_{\mathrm{i}}\right)+\mathrm{u}_{0 \mathrm{j}} \\
& \beta_{1 \mathrm{j}}=\gamma_{10}+\beta_{11} *\left(\text { Group }_{\mathrm{i}}\right)+\beta_{12} *\left(\text { Attachment index }_{\mathrm{i}}\right) \\
& +\beta_{13} *\left(\text { Interaction }_{\mathrm{i}}\right)+\mathrm{u}_{1 \mathrm{j}} \\
& \beta_{2 \mathrm{j}}=\gamma_{20}+\beta_{21} *\left(\text { Group }_{\mathrm{i}}\right)+\beta_{22} *\left(\text { Attachment index }_{\mathrm{i}}\right) \\
& \left.+\beta_{23} * \text { Interaction }_{\mathrm{i}}\right)+\mathrm{u}_{2 \mathrm{j}}
\end{aligned}
$$

Journal of Pain Research

\section{Publish your work in this journal}

The Journal of Pain Research is an international, peer reviewed, open access, online journal that welcomes laboratory and clinical findings in the fields of pain research and the prevention and management of pain. Original research, reviews, symposium reports, hypothesis formation and commentaries are all considered for publication.

\section{Dovepress}

The manuscript management system is completely online and includes a very quick and fair peer-review system, which is all easy to use. Visit http://www.dovepress.com/testimonials.php to read real quotes from published authors. 